\title{
Correction to: Longitudinal multi-modal muscle-based biomarker assessment in motor neuron disease
}

Thomas M. Jenkins ${ }^{1,2}$ - James J. P. Alix ${ }^{1,3}$ - Jacob Fingret ${ }^{1} \cdot$ Taniya Esmail $^{1} \cdot$ Nigel Hoggard $^{4} \cdot$ Kathleen Baster $^{5}$. Christopher J. McDermott ${ }^{1,2} \cdot$ lain D. Wilkinson ${ }^{4} \cdot$ Pamela J. Shaw ${ }^{1,2}$

Published online: 28 November 2019

(c) The Author(s) 2019

\section{Correction to: Journal of Neurology}

https://doi.org/10.1007/s00415-019-09580-x

The original version of this article unfortunately contained a mistake. The numbers in the Table 5 appear to have been reproduced wrongly as dates, rather than percentages.

The corrected Table 5 is given in the next page.

Open Access This article is distributed under the terms of the Creative Commons Attribution 4.0 International License (http://creativeco mmons.org/licenses/by/4.0/), which permits unrestricted use, distribution, and reproduction in any medium, provided you give appropriate credit to the original author(s) and the source, provide a link to the Creative Commons license, and indicate if changes were made.

The original article can be found online at https://doi.org/10.1007/ s00415-019-09580-x.

Thomas M. Jenkins

t.m.jenkins@sheffield.ac.uk

1 Sheffield Institute for Translational Neuroscience, University of Sheffield, 385a Glossop Road, Sheffield S10 2HQ, UK

2 Department of Neurology, Sheffield Teaching Hospitals NHS Foundation Trust, Sheffield, UK

3 Departments of Neurophysiology, Sheffield Teaching Hospitals NHS Foundation Trust, Sheffield, UK

4 Academic Unit of Radiology, University of Sheffield, Sheffield, UK

5 Statistics Services Unit, School of Mathematics and Statistics, University of Sheffield, Sheffield, UK 
Table 5 Longitudinal radiological changes in relative T2-signal in MND patients

\begin{tabular}{|c|c|c|c|c|c|c|}
\hline Muscle & $\begin{array}{l}\text { Mean } \% \text { change in relative } \\
\text { T2-signal between baseline and } \\
4 \text { months }(95 \% \mathrm{CI})\end{array}$ & $P$ value & $\begin{array}{l}\text { Mean } \% \text { change in relative } \\
\text { T2-signal between baseline and } \\
12 \text { months }(95 \% \mathrm{CI})\end{array}$ & $P$ value & SRM & Var ratio \\
\hline Tongue & $-4.8(-11.2,1.5)$ & 0.123 & $-2.4(-10.2,5.4)$ & 0.540 & -0.08 & 0.66 \\
\hline Right trapezius & $-6.9(-19.1,5.2)$ & 0.251 & $0.2(-14.5,14.8)$ & 0.981 & 0.06 & 0.17 \\
\hline Left trapezius & $-10.9(-22.9,0.9)$ & 0.066 & $-1.7(-16.1,12.8)$ & 0.816 & -0.02 & 0.17 \\
\hline Right sternocleidomastoid & $-3.7(-13.4,6.0)$ & 0.443 & $0.3(-11.5,12.2)$ & 0.949 & 0.10 & 0.43 \\
\hline Left sternocleidomastoid & $-4.4(-14.5,5.6)$ & 0.376 & $-4.1(-16.3,8.2)$ & 0.502 & -0.11 & 0.37 \\
\hline Splenius capitis & $4.2(-4.3,12.8)$ & 0.321 & $-5.4(-16.0,5.1)$ & 0.297 & -0.42 & 0.47 \\
\hline Right deltoid & $2.7(-5.0,10.4)$ & 0.486 & $-4.8(-14.4,4.7)$ & 0.304 & -0.27 & 0.71 \\
\hline Left deltoid & $1.9(-6.6,10.4)$ & 0.654 & $4.6(-5.8,15.2)$ & 0.365 & 0.08 & 0.59 \\
\hline Right biceps brachii & $-2.2(-13.8,9.4)$ & 0.700 & $11.3(-2.9,25.7)$ & 0.107 & 0.18 & 0.55 \\
\hline Left biceps brachii & $-3.5(-17.4,10.1)$ & 0.597 & $-1.9(-18.6,14.7)$ & 0.814 & -0.21 & 0.26 \\
\hline Right triceps & $-13.9(-47.2,18.0)$ & 0.378 & $-22.3(-65.1,18.4)$ & 0.266 & -0.19 & 0.10 \\
\hline Left triceps & $-0.0(-31.3,31.2)$ & 0.998 & $-0.0(-22.1,22.1)$ & 0.708 & -0.18 & 0.00 \\
\hline Right brachioradialis & $7.3(-15.0,29.9)$ & 0.507 & $10.4(-15.4,36.8)$ & 0.414 & 0.04 & 0.06 \\
\hline Left brachioradialis & $7.0(-16.8,31.2)$ & 0.551 & $9.9(-19.3,39.6)$ & 0.492 & 0.82 & 0.23 \\
\hline Right first dorsal interosseous & $18.4(-1.8,39.8)$ & 0.067 & $31.1(6.8,57.4)$ & 0.010 & 0.64 & 0.43 \\
\hline Left first dorsal interosseous & $-0.0(-26.2,24.6)$ & 0.952 & $16.1(-16.2,50.4)$ & 0.311 & 0.97 & 0.41 \\
\hline Right thenar & $14.4(-7.1,36.6)$ & 0.177 & $21.4(-4.9,48.9)$ & 0.100 & 0.76 & 0.26 \\
\hline Left thenar & $-8.7(-42.1,23.8)$ & 0.586 & $-10.0(-50.1,29.8)$ & 0.607 & -0.57 & 0.17 \\
\hline Right hypothenar & $5.5(-10.6,22.0)$ & 0.491 & $13.8(-5.8,34.4)$ & 0.155 & 0.27 & 0.73 \\
\hline Left hypothenar & $3.1(-12.6,18.8)$ & 0.691 & $2.3(-16.2,20.9)$ & 0.802 & 0.22 & 0.34 \\
\hline Right thoracic paraspinal & $-1.0(-4.4,2.4)$ & 0.903 & $16.7(-3.0,37.6)$ & 0.087 & 0.30 & 0.69 \\
\hline Left thoracic paraspinal & $1.6(-12.1,15.5)$ & 0.811 & $4.2(-12.7,21.4)$ & 0.610 & 0.24 & 0.70 \\
\hline Right psoas & $6.2(-19.9,33.1)$ & 0.627 & $35.7(4.6,71.1)$ & 0.020 & 0.53 & 0.52 \\
\hline Left psoas & $-11.8(-42.8,17.9)$ & 0.419 & $-15.7(-54.1,20.9)$ & 0.382 & -0.01 & 0.31 \\
\hline Right gluteus maximus & $-3.0(-24.3,18.1)$ & 0.774 & $22.0(-3.1,48.8)$ & 0.076 & 0.44 & 0.49 \\
\hline Left gluteus maximus & $-14.2(-42.3,12.2)$ & 0.277 & $-14.8(-49.8,18.4)$ & 0.363 & -0.10 & 0.53 \\
\hline Right quadriceps & $5.4(-2.5,13.6)$ & 0.168 & $18.8(9.0,29.6)$ & $<0.001 *$ & 0.86 & 0.90 \\
\hline Left quadriceps & $1.2(-7.4,9.7)$ & 0.784 & $11.4(2.9,20.6)$ & 0.028 & 0.39 & 0.91 \\
\hline Right hamstrings & $-2.2(-10.2,5.6)$ & 0.564 & $17.4(7.7,28.2)$ & $<0.001 *$ & 0.98 & 0.93 \\
\hline Left hamstrings & $1.9(-7.1,11.0)$ & 0.666 & $14.4(3.3,26.3)$ & $0.008 *$ & 0.65 & 0.89 \\
\hline Right tibialis anterior & $10.7(0.8,21.2)$ & $\mathbf{0 . 0 3 0}$ & $28.5(15.9,42.7)$ & $<0.001 *$ & 0.82 & 0.87 \\
\hline Left tibialis anterior & $4.1(-7.9,16.5)$ & 0.180 & $20.9(6.0,37.2)$ & $0.004 *$ & 0.33 & 0.86 \\
\hline Right gastrocnemius/soleus & $5.6(-3.2,14.7)$ & 0.200 & $18.9(8.0,30.6)$ & $<0.001 *$ & 0.64 & 0.83 \\
\hline Left gastrocnemius/soleus & $0.5(-8.2,9.1)$ & 0.911 & $18.7(8.0,30.3)$ & $<0.001 *$ & 0.78 & 0.88 \\
\hline Muscle-of-onset & $-0.08(-16.1,16.0)$ & 0.698 & $16.6(-2.9,37.3)$ & 0.070 & 0.77 & 0.73 \\
\hline Region-of-onset & $2.7(-4.3,9.7)$ & 0.437 & $14.1(5.5,23.2)$ & $0.001 *$ & 0.87 & 0.89 \\
\hline Summary mean & $-0.1(-6.2,5.9)$ & 0.972 & $8.8(1.3,16.4)$ & 0.017 & 0.36 & 0.56 \\
\hline
\end{tabular}

Significant T2-weighted radiological changes in MND patients between baseline and subsequent 4 and 12 month time-points are highlighted in bold. Results surviving multiple comparisons correction are asterisked

CI confidence interval, SRM standardized response mean, Var variance 